\title{
Targeting the miR-630/YAP1/ERK feedback loop in epidermal growth factor receptor-mutated lung adenocarcinomas
}

\author{
Jinglin Zhang, Alfred S. L. Cheng, Jun Yu, Ka Fai To, Wei Kang \\ Department of Anatomical and Cellular Pathology, State Key Laboratory of Translational Oncology, State Key laboratory of Digestive Diseases, The \\ Chinese University of Hong Kong, Hong Kong, China \\ Correspondence to: Professor Wei Kang. Department of Anatomical and Cellular Pathology, Prince of Wales Hospital, The Chinese University of \\ Hong Kong, Hong Kong, China. Email: weikang@cuhk.edu.hk; Professor Ka Fai To. Department of Anatomical and Cellular Pathology, Prince of \\ Wales Hospital, The Chinese University of Hong Kong, Hong Kong, China. Email: kfto@cuhk.edu.hk. \\ Provenance: This is an invited Editorial commissioned by the Section Editor Chunlin Ou (Cancer Research Institute of Central South University, \\ Changsha, China). \\ Comment on: Wu DW, Wang YC, Wang L, et al. A low microRNA-630 expression confers resistance to tyrosine kinase inhibitors in EGFR-mutated \\ lung adenocarcinomas via miR-630/YAP1/ERK feedback loop. Theranostics 2018;8:1256-69.
}

Submitted Sep 02, 2018. Accepted for publication Sep 26, 2018.

doi: $10.21037 /$ jtd.2018.10.16

View this article at: http://dx.doi.org/10.21037/jtd.2018.10.16

Lung cancer is the most prevalent cancer type globally. Over 1.8 million new cases are diagnosed and 1.6 million deaths are reported yearly. The 5 -year survival rates of lung cancer vary in different disease stages, histological types, genders and regions. Early screening, detection and treatment are helpful to improve survival rates (1). In the last few years, targeted therapy is becoming a promising strategy for advanced lung cancer patients. In general, targeted therapies include tyrosine kinase inhibitors (TKI) of active epidermal growth factor receptor (EGFR) mutations, anaplastic lymphoma kinase (ALK) rearrangement or angiogenesis, as well as some monoclonal antibodies for vascular endothelial growth factor (VEGF) (2). In recent issue of Theranostics, Wu et al. reported one of the molecular mechanisms for drug resistance (3). They unraveled a comprehensive regulatory loop of EGFR-TKI resistance in lung carcinoma by in vitro and in vivo studies together with validation from primary samples. Their findings came up with a novel therapeutic direction for lung cancer patients with EGFR mutations.

\section{TKI therapy for EGFR mutation in lung carcinoma}

Concerning to the variations in clinical characteristics, histological features and response to treatment, lung cancer is subgrouped as two main classes, $10-15 \%$ as small cell lung carcinomas (SCLC) and $80-85 \%$ as non-small cell carcinomas (NSCLC). In current treatment guidelines and standards, surgery is recommended for NSCLC, and chemotherapy and/or radiotherapy are more eligible for SCLC (4). As the emergence of driver mutations, targeted therapy against specific oncogenic mutation has been developed in preclinical trials and clinical use. Great progress has been made in diagnosis and treatment (5). EGFR is known as a receptor tyrosine kinase. The EGFRactivating mutants make up a major class in NSCLC. TKIs against EGFR mutants have therefore been developed and applied for patients. Notably, patients harboring the EGFRexon 19 frame deletions and the exon 21 L858A single point mutation are found most sensitive to TKI treatment. The first-generation TKI such as gefitinib and erlotinib $(6,7)$, and the second-generation TKI afatinib are considered as a standard TKI therapy (5). These targeting TKIs not only increase response rates, but also improve better overall survival. Despite of the favorable initial outcomes, the disease recurrence is frequently observed in a large number of patients. The main reason is TKI drug resistance. About $50 \%$ of NSCLC patients with TKI resistance are identified as T790M mutation positive. The TKI targeted therapy for these patients is still facing lots of limitations such as toxicity and other adverse effects (8). Hence, the molecular 
Table 1 miRNA list involved in lung adenocarcinoma TKI resistance through apoptosis regulation

\begin{tabular}{lcl}
\hline miRNA & Expression & Putative targets and mechanisms involved in TKI resistance \\
\hline miR-21 & Up & Targeting PTEN and PDCD4, and inhibiting apoptosis (12) \\
miR-19b & Up & $\begin{array}{l}\text { Enhancing apoptosis resistance by targeting PP2A and BIM (13) } \\
\text { miR-221/222 }\end{array}$ \\
Up & $\begin{array}{l}\text { Targeting multiple genes, such as TRAIL, CDKN1B, PTEN, TIMP3, PUMA, BIM, and APAF1, thus to inhibit } \\
\text { apoptosis (14) }\end{array}$ \\
miR-200 & Down & $\begin{array}{l}\text { Targeting MIG6 to induce cell apoptosis (15) } \\
\text { miR-451 }\end{array}$ \\
miR-34a & Down & Downregulating RAB14 and increasing the expression of BAX or Bad, thus enhancing apoptosis (16) \\
\hline
\end{tabular}

TKI, tyrosine kinase inhibitors.

mechanisms for acquired resistance of TKIs remain to be clarified. Wu et al. thus provided novel molecular evidence to indicate miR-630/YAP1/ERK regulatory feedback loop which might contribute to TKI resistance in NSCLC patients with EGFR mutation (3). They also proposed that combination of TKI and other targeted inhibitors might ameliorate the TKI resistance effect and reduce tumor recurrence rate in EGFR-mutated lung adenocarcinoma patients.

\section{Low miR-630 confers to TKI resistance in lung carcinoma}

MicroRNAs (miRNAs) are non-coding endogenous RNAs that can recognize and bind to the $3^{\prime}$ untranslated region (3' UTR) of targeted mRNA, thus to repress the expression of the targeted genes (9). Accumulating number of studies have demonstrated the multifunctional roles of miRNAs during the initiation and progression of lung carcinomas. The specific signatures discovered by miRNA profiling have been applied in both early diagnosis and prognostic prediction. miRNAs also serve as potential biomarkers and therapeutic targets for cancers (10). Because of the low degradation in formalin-fixed paraffin-embedded (FFPE) samples and existence in body fluids, miRNAs acquire much interest in lung cancer research as non-invasive biomarkers (11). During the past few years, the dysregulated miRNAs, which were associated with TKI resistance, have been identified in EGFR-mutation lung adenocarcinoma. Among these altered miRNAs, some of them induces resistance through regulation of apoptosis related genes. For example, upregulated miR-21 (12), miR-19b (13), and miR-221/222 (14), or downregulated miR-200 (15), miR-451 (16), and miR34a (17), were strongly correlated with TKI resistance through targeting multiple apoptosis related genes (Table 1). In this study, the authors examined miR-630 expression in six lung adenocarcinoma cell lines with EGFR mutation (3). They detected high expression of miR-630 in gefitinibsensitive cells, while gefitinib-resistant cells showed relative low expression. The result implied the correlation between miR-630 and gefitinib responses in EGFR-mutated lung adenocarcinoma. Interestingly, in a gefitinib responsive cell line, miR-630 was increased after gefitinib treatment. Their findings established that miR-630 is strongly associated with TKI-resistant therapy. By treating with gefitinib, the gefitinib-resistant cells exhibited a relative stable level of pERK and pAKT, which led to the increase binding activity of SP1 to miR-630 promoter thus to inhibit the expression of miR-630. It clearly elucidated how miR-630 was involved in TKI resistance and exhibited therapeutic potential in treating TKI-resistant cases.

\section{Hippo pathway and TKI therapy in lung carcinogenesis}

The key components of Hippo signaling such as MST1/2, LATS1/2 and YAP1/TAZ are involved in lung tumorigenesis to promote cancer cell proliferation and suppress apoptosis (18-20). YAP1 is known as the functional downstream effector of Hippo signaling. YAP1 not only displays oncogenic function, but also confers to TKI resistance. In NSCLC patients, aberrant increase of YAP1 was detected and activated YAP1 was significantly associated with poor prognosis (21). A recent study reported that the YAP1-mediated signaling was regulated by EGFR related crosstalk in hepatocellular carcinoma (HCC), meanwhile YAP1 inhibition by inhibitors enhanced the EGFR-TKI treatment response (22). The upregulation 
of YAP1 was observed in lung cancer cell lines which acquired gefitinib-resistance and overexpression of YAP1 induced EGFR-TKI resistance. Moreover, inhibiting YAP1 improved the sensitivity of EGFR-TKI drugs in lung adenocarcinoma with primary or acquired EGFRTKI resistance (23). Similarly, Wu et al. reported higher expression of YAP1 was presented in lung cancer cell lines with gefitinib-resistance compared to the parental cell lines without TKI resistance (3). Since YAP1 was a potential target of miR-630 (24), they tried to reveal the correlative regulation between miR-630 and YAP1 in the EGFR-TKI resistance of lung carcinogenesis. As shown in their results, YAP1 was increased by inhibiting miR-630, while miR630 overexpression suppressed the expression of YAP1. Thereby, YAP1 was proved to be the most important target of miR-630 driving EGFR-TKI resistance. Further, they confirmed that the function of miR-630/YAP1 was mainly mediated by ERK signaling instead of AKT signaling. The authors highlighted the significant regulatory loop of miR630/YAP1/ERK contributing to EGFR-TKI resistance in lung adenocarcinoma.

\section{Decreased apoptosis also gives rise to EGFR- TKI resistance}

Inducing apoptosis is one of the major strategies for antitumor therapy and anti-apoptosis effects do exist in TKIresistance. Sordella et al. previously reported that EGFRmutated NSCLC cells activated AKT and STAT signaling pathways to resist apoptosis and facilitate cell survival. Thus, the cancer cells were much resistant to conventional chemotherapeutic drugs. However, targeting mutated EGFR, or using pharmacological inhibitors of AKT and STAT, dramatically induced expanding apoptosis and reversed the drug resistance NSCLC cells with EGFR mutation (25). In recent years, miRNAs have been demonstrated as promising therapeutic targets against tumorigenesis and TKI resistance. A study indicated the miR-21 overexpression, regulated by EGFR signaling, contributed to lung carcinogenesis through inhibiting apoptosis. To identify the apoptosis-related factors involved in miR-630/YAP1/ERK mediated EGFR-TKI resistance, the authors performed a chip assay to screen which protein dominantly increased after miR-630 overexpression. The Bcl-2-associated death (BAD) promoter, a pro-apoptotic member involves in the beginning of apoptosis, was revealed (3). When re-overexpressing miR-630 in TKI resistance cells, the level of Bad was restored and apoptotic response was stimulated. Integrating their results, suppression of miR-630 and degradation of Bad were correlated with the persistent increase of the YAP1 and activation of ERK. This molecular mechanism was proposed to mediate the TKI resistance in lung carcinoma with EGFR mutation. Targeting the regulatory miR-630/ YAP1/ERK loop might have clinical implication.

\section{Summary and future direction}

With comprehensive evidences, the authors elucidated a potent regulatory cascade that confers to lung adenocarcinoma cells harboring EGFR mutation. In this study, down-regulation of miR-630 was screened in TKI resistant cells. Its decrease led to the activation of YAP1 and ERK signaling. Conversely, the intense binding of SP1, an ERK dependent transcription factor, suppressed miR630 expression and the pro-apoptotic protein Bad. The miR-630/YAP1/ERK feedback loop, therefore, reduced cell apoptosis and induced TKI resistance persistently. To summarize this study, reduced expression of miR-630 is a trigger in the feedback loop contributing to TKI resistance. Upregulation of YAP1 and activation of ERK signaling were further delineated how lung cancer cells resist TKI treatment and devoid apoptosis.

Targeting this feedback loop hints a novel direction for reversing TKI resistance. Combining administration of the YAP1 inhibitor VP, or the ERK inhibitor AZD6244, together with the TKI might resolve the problem in tumor recurrence. This combinational therapeutic strategy sheds new light on the EGFR-mutated lung adenocarcinoma patients who are resistant to TKI therapy.

\section{Acknowledgement}

Funding: Supported by grants from the Research Grants Council of the Hong Kong Special Administrative Region, China [Project No.: CUHK 14110016 and 14118518 (for GRF projects)] and CUHK Direct Grant for Research (2017.001). We also acknowledge the support from Core Utilities of Cancer Genomics and Pathobiology of Department of Anatomical and Cellular Pathology, The Chinese University of Hong Kong.

\section{Footnote}

Conflicts of Interest: The authors have no conflicts of interest to declare. 


\section{References}

1. Hirsch FR, Scagliotti GV, Mulshine JL, et al. Lung cancer: current therapies and new targeted treatments. Lancet 2017;389:299-311.

2. Sculier JP, Berghmans T, Meert AP. Advances in target therapy in lung cancer. Eur Respir Rev 2015;24:23-9.

3. Wu DW, Wang YC, Wang L, et al. A low microRNA-630 expression confers resistance to tyrosine kinase inhibitors in EGFR-mutated lung adenocarcinomas via miR-630/ YAP1/ERK feedback loop. Theranostics 2018;8:1256-69.

4. Pisters KM, Evans WK, Azzoli CG, et al. Cancer Care Ontario and American Society of Clinical Oncology adjuvant chemotherapy and adjuvant radiation therapy for stages I-IIIA resectable non small-cell lung cancer guideline. J Clin Oncol 2007;25:5506-18.

5. Kris MG, Johnson BE, Berry LD, et al. Using multiplexed assays of oncogenic drivers in lung cancers to select targeted drugs. JAMA 2014;311:1998-2006.

6. Lynch TJ, Bell DW, Sordella R, et al. Activating mutations in the epidermal growth factor receptor underlying responsiveness of non-small-cell lung cancer to gefitinib. N Engl J Med 2004;350:2129-39.

7. Shepherd FA, Rodrigues Pereira J, Ciuleanu T, et al. Erlotinib in previously treated non-small-cell lung cancer. N Engl J Med 2005;353:123-32.

8. Mok TS, Wu YL, Ahn MJ, et al. Osimertinib or PlatinumPemetrexed in EGFR T790M-Positive Lung Cancer. N Engl J Med 2017;376:629-40.

9. Doench JG, Sharp PA. Specificity of microRNA target selection in translational repression. Genes Dev 2004;18:504-11.

10. Inamura K, Ishikawa Y. MicroRNA In Lung Cancer: Novel Biomarkers and Potential Tools for Treatment. J Clin Med 2016;5:36.

11. Inamura K. Diagnostic and Therapeutic Potential of MicroRNAs in Lung Cancer. Cancers (Basel) 2017;9.

12. Li B, Ren S, Li X, et al. MiR-21 overexpression is associated with acquired resistance of EGFR-TKI in nonsmall cell lung cancer. Lung Cancer 2014;83:146-53.

13. Baumgartner U, Berger F, Hashemi Gheinani A, et al. miR-19b enhances proliferation and apoptosis resistance via the EGFR signaling pathway by targeting PP2A and BIM in non-small cell lung cancer. Mol Cancer 2018;17:44.

14. Garofalo M, Romano G, Di Leva G, et al. EGFR and MET receptor tyrosine kinase-altered microRNA expression induces tumorigenesis and gefitinib resistance in lung cancers. Nat Med 2011;18:74-82.

15. Izumchenko E, Chang X, Michailidi C, et al. The TGFbeta-miR200-MIG6 pathway orchestrates the EMTassociated kinase switch that induces resistance to EGFR inhibitors. Cancer Res 2014;74:3995-4005.

16. Wang R, Wang ZX, Yang JS, et al. MicroRNA-451 functions as a tumor suppressor in human non-small cell lung cancer by targeting ras-related protein 14 (RAB14). Oncogene 2011;30:2644-58.

17. Zhou JY, Chen X, Zhao J, et al. MicroRNA-34a overcomes HGF-mediated gefitinib resistance in EGFR mutant lung cancer cells partly by targeting MET. Cancer Lett 2014;351:265-71.

18. Valero V, 3rd, Pawlik TM, Anders RA. Emerging role of Hpo signaling and YAP in hepatocellular carcinoma. J Hepatocell Carcinoma 2015;2:69-78.

19. Teoh SL, Das S. The Emerging Role of the Hippo Pathway in Lung Cancers: Clinical Implications. Curr Drug Targets 2017;18:1880-92.

20. Kang W, Cheng AS, Yu J, et al. Emerging role of Hippo pathway in gastric and other gastrointestinal cancers. World J Gastroenterol 2016;22:1279-88.

21. Wang Y, Dong Q, Zhang Q, et al. Overexpression of yes-associated protein contributes to progression and poor prognosis of non-small-cell lung cancer. Cancer Sci 2010;101:1279-85.

22. Xia H, Dai X, Yu H, et al. EGFR-PI3K-PDK1 pathway regulates YAP signaling in hepatocellular carcinoma: the mechanism and its implications in targeted therapy. Cell Death Dis 2018;9:269.

23. Lee JE, Park HS, Lee D, et al. Hippo pathway effector YAP inhibition restores the sensitivity of EGFR-TKI in lung adenocarcinoma having primary or acquired EGFR-TKI resistance. Biochem Biophys Res Commun 2016;474:154-60.

24. Huang Y, Chuang A, Hao H, et al. Phospho-DeltaNp63alpha is a key regulator of the cisplatin-induced microRNAome in cancer cells. Cell Death Differ 2011;18:1220-30.

25. Sordella R, Bell DW, Haber DA, et al. Gefitinibsensitizing EGFR mutations in lung cancer activate antiapoptotic pathways. Science 2004;305:1163-7.

Cite this article as: Zhang J, Cheng AS, Yu J, To KF, Kang W. Targeting the miR-630/YAP1/ERK feedback loop in epidermal growth factor receptor-mutated lung adenocarcinomas. J Thorac Dis 2018;10(Suppl 33):S4017-S4020. doi: 10.21037/ jtd.2018.10.16 